\title{
Evaluation of Pattern Recognition Algorithms for Applications on Power Factor Compensation
}

\author{
Alexandre C. Moreira ${ }^{1}$ (D) Helmo K. M. Paredes ${ }^{2}$ (D) - Wesley A. de Souza ${ }^{3}$ (D) Pedro H. J. Nardelli ${ }^{4}$ (I) \\ Fernando P. Marafão ${ }^{2}$ (D) . Luiz C. P. da Silva ${ }^{3}$
}

Received: 29 June 2017 / Revised: 18 October 2017 / Accepted: 18 November 2017 / Published online: 5 December 2017

(c) Brazilian Society for Automatics-SBA 2017

\begin{abstract}
This paper assesses different applied pattern recognition algorithms to decide the most appropriate power factor compensator for a particular point of common coupling. Power factor, current unbalance factor, total demand distortion, voltage harmonic distortion and reactive power daily variation, as well as human expertise, are the key parameters used to set each recognition algorithm. These algorithms are then trained with a series of both simulation and experimental data. Numerical results consistently indicate the decision-tree algorithm with depth 20 as the best classifier for power factor improvement in terms of all metrics considered in this work.
\end{abstract}

Keywords Active compensators · Passive compensators · Pattern recognition $\cdot$ Power factor $\cdot$ Reactive and harmonic compensation

Alexandre C. Moreira

amoreira@ufsj.edu.br

Helmo K. M. Paredes

hmorales@sorocaba.unesp.br

Wesley A. de Souza

wesley@fc.unesp.br

Pedro H. J. Nardelli

nardelli@ee.oulu.fi

Fernando P. Marafão

fmarafao@sorocaba.unesp.br

Luiz C. P. da Silva

lui@dsee.fee.unicamp.br

1 Telecommunications and Mechatronic Engineering Department (DETEM), Federal University of São João del-Rei (UFSJ), Rodovia MG 443, KM 7, Ouro Branco, MG 36420-000, Brazil

2 Institute of Science and Technology, São Paulo State University (Unesp), Av. Três de Março, 511, Sorocaba, SP 18087-180, Brazil

3 Department of Energy and Systems (DSE), School of Electrical and Computer Engineering (FEEC), University of Campinas (UNICAMP), Av. Albert Einstein, 400, Campinas, SP 13083-970, Brazil

4 Centre for Wireless Communications (CWC), University of Oulu, Erkki Koiso-Kanttilan katu 3, 90570 Oulu, Finland

\section{Introduction}

The increase in nonlinear loads, the severity of harmonic pollution, and the load unbalance in electrical power systems have attracted the attention of power system engineers to develop proper solutions for modern electrical power systems. Some examples of industrial nonlinear loads that might generate harmonic currents or load unbalance are: arc furnace, induction furnace, adjustable-speed drives (ASDs), welding machines, and power electronic loads.

Regarding the harmonic currents, it is known that the flow through the system impedance results in nonsinusoidal voltage drops, which can affect the grid voltage quality (Das 2015; IEEE P519 2015). In three-phase systems, harmonics can also cause current unbalance and excessive neutral currents. Consequently, the injected harmonics, reactive power, unbalance, and excessive neutral currents may decrease the system efficiency and the power factor (IEEE 2010; Tenti et al. 2011). They may also cause severe disturbances to other consumers. Thus, this subject has become extremely important regarding power quality (PQ) aspects, revenue metering, and measurement issues (IEEE 2010).

Different from the reactive power drawn by a linear balanced load, the harmonic currents cannot be easily compensated by the use of capacitors and inductors. In this case, passive or active harmonic filters are usually required 
to minimize harmonic and reactive power flow. Moreover, if unbalanced three-phase loads are considered, the definition of the compensator may be even more sophisticated.

In this scenario, the decision of which compensator should be used is generally based on the expertise of the power system engineers. By means of careful analysis and simulations, these specialists might indicate proper solutions in order to mitigate the disturbances and control PQ indices according to the standards limits. Thus, the specialist's knowledge plays a critical role in the decision-making process. This professional, however, needs to deal with an enormous amount of data. In such context, the development of automatic analytic tools becomes very important for evaluating and identifying several PQ disturbances and their respective suitable solution.

Indeed, the growing number of pattern recognition applications in the last few decades is noteworthy, specially due to the increasing need for automation in different segments of society. This demand has been met by the evolution of digital processors, signal processing techniques, as well as the amount of digital information on the Internet. Applications of pattern recognition can be found in machine vision, robotics, biometrics, information retrieval, and instrumentation, likewise in power systems (Ribeiro et al. 2013; Bollen and $\mathrm{Gu} 2006$ ).

Several researchers have published power system applications based on pattern recognition algorithms (PRAs) (Samantaray 2013; Jamehbozorg and Shahrtash 2010; Samantaray 2009; Galil et al. 2004; Biet 2013; Gaouda et al. 2002, 2001; Moravej et al. 2015; Livani and Evrenosoglu 2013; Cerqueira et al. 2008; Axelberg et al. 2007; Rodriguez et al. 2014; Monedero et al. 2007; Hajian and Foroud 2014; Lieberman et al. 2011). For example, different classification methods such as decision tree (DT) (Samantaray 2013; Jamehbozorg and Shahrtash 2010; Samantaray 2009; Galil et al. 2004; Barbosa and Ferreira 2013), $k$-nearest neighbor ( $K$-NN) (Biet 2013; Gaouda et al. 2002, 2001), support vector machines (SVMs) (Moravej et al. 2015; Livani and Evrenosoglu 2013; Cerqueira et al. 2008; Axelberg et al. 2007), and different types of artificial neural networks (ANNs) (Gaouda et al. 2001; Rodriguez et al. 2014; Monedero et al. 2007; Andreoli et al. 2013) have been developed for different PQ applications. However, for power factor improvement, this approach is still missing. The choice of a suitable classifier in this case is very important to avoid a bad classification, resulting in an inappropriate compensator, which could be less effective than other potential options or could lead to unstable conditions.

Therefore, assuming the use of PRA, the aim of this paper is to determine the most suitable classifier for the development of an automatic system, which may be able to indicate the most appropriate power factor compensator for a certain point of common coupling.

Following, Sect. 2 presents some examples of pattern recognition applications in power systems. In Sect. 3, the authors present the main characteristics of the proposed methodology to evaluate different PRAs regarding the problem of power factor improvement. Section 4 presents the performance indices used for classifiers evaluation. In Sect. 5, the authors compare the classification results by means of computational simulations. Experimental results are discussed in Sect. 6, while the main conclusions are described in Sect. 7.

\section{Pattern Recognition Applications in Power Systems}

Pattern recognition techniques are automated tools for decision-making processes (Ribeiro et al. 2013; Bollen and $\mathrm{Gu} 2006$ ). In recent years, pattern recognition methods have been widely used in power systems. Examples in power systems include fault analysis (Jamehbozorg and Shahrtash 2010; Moravej et al. 2015; Livani and Evrenosoglu 2013), power quality (Galil et al. 2004; Gaouda et al. 2001; Cerqueira et al. 2008; Axelberg et al. 2007; Rodriguez et al. 2014; Monedero et al. 2007; Hajian and Foroud 2014; Upadhyaya et al. 2015), electrical machines (Biet 2013; Junior et al. 2014; Palácios et al. 2016; Santana et al. 2017), and flexible AC transmission systems (FACTS) (Samantaray 2013). Pattern recognition provides tremendous flexibility benefits. This section provides some of the theoretical concepts that will be referred to in subsequent sections. Examples and strategies will be given to support the process of adopting pattern recognition in the context of power systems.

\subsection{Decision Tree Classifier}

Decision tree is a data structure consisting of a root node, decision nodes, and leaf nodes. The DT algorithm allows for simplification by pruning techniques, which reduce the size of the tree according to a user-defined level. The algorithm can produce either DT or rules in the form of: "if," "then" or "else." The advantage of this proposal is the representation, which is more understandable than other methods like neural networks (NNs) (Galil et al. 2004). However, DT has the disadvantage of only working with linearly separable data.

Recently, DT has been found highly successful for power system applications as FACTS (Samantaray 2013), fault classification (Jamehbozorg and Shahrtash 2010; Samantaray 2009) and power quality (Galil et al. 2004). In (Samantaray 2013), the authors presented a data-mining model for 
fault-zone identification of a flexible AC transmission system. Moreover, a comparison between DT and SVM, which will be discussed later on, is performed for this kind of problem.

In (Jamehbozorg and Shahrtash 2010), researchers consider fault classification in single-circuit transmission lines in which conventional methods as mathematical operations on voltages and current could not classify the faults. By means of DT, however, the authors showed that the proposed method could classify the faults with reasonable accuracy. In (Samantaray 2009), a new approach for fault-zone classification on transmission lines equipped with thyristor-controlled series compensator (TCSC) and unified power flow controller (UPFC) using DT is presented. The authors showed that the processing time of SVM is higher compared to the proposed DT.

In (Galil et al. 2004), DT has been utilized for the classification of PQ. The wavelet transform is utilized to produce representative attribute vectors. The DT results for classification are compared with the artificial neural networks' results.

\subsection{K-Nearest Neighbor Classifier}

$K$-nearest neighbor $(K-\mathrm{NN})$ is a supervised learning algorithm that finds the first $\mathrm{K}$ minimum distances' points, and the decision is made based on the group that has the majority minimum distances. It can produce any arbitrary complex surface to separate the classes based on the configuration of the sample points and their distance relations to each other. The closeness is usually measured in terms of Euclidean distances.

The literature is very rich with solutions that use the $K$ NN pattern recognition technique. Regarding power system applications (Biet 2013), $K$-NN was applied to a diagnosis method to find rotor faults. In (Gaouda et al. 2002), $K$-NN is applied to classify different power quality disturbances such as sags, swell, and harmonics. The wavelet transform and multiresolution signal decomposition are used for extracting attributes from different distorted signals.

The main advantage of $K$-NN in comparison with other algorithms is its good classification accuracy for mixed power quality problems (Gaouda et al. 2001). On the other hand, $K$-NN is computationally expensive in the search for the $K$ nearest neighbors, mainly when the data set is very large. In addition, $K$-NN may provide inaccurate results for highdimensional spaces (more than 10-15 dimensions) (Beyer et al. 1999).

\subsection{Support Vector Machines Classifier}

Cortes and Vapnik introduced SVM as a pattern recognition method. This supervised learning technique can be applied to either classification or regression. When it is used for pattern recognition, it searches a hyperplane that completely separates a data set. SVMs can also extend this to a nonlinear decision boundary using the kernel method.

Applications within power systems using SVM are, for instance, fault analysis or classification (Moravej et al. 2015; Livani and Evrenosoglu 2013), power quality event classification (Cerqueira et al. 2008), and voltage disturbances classification (Axelberg et al. 2007). In (Moravej et al. 2015), the authors presented the limitation of some distance relay functions in the presence of UPFC. The SVM classifier is employed for fault-type identification, fault-loop status supervision, and fault-zone detection.

In (Livani and Evrenosoglu 2013), the wavelet transform is utilized to extract transient information from the recorded voltages, and SVM is used to classify the fault type in the transmission networks. In (Cerqueira et al. 2008), the authors presented two versions of a novel classification method for power quality event classification based on SVM. In (Axelberg et al. 2007), the authors suggested a SVM classification system for voltage disturbances such as fault or transformer energizing.

The SVMs based on the decision boundary margin maximization have better generalization ability compared to DT and neural network (NN). Besides, the SVMs do not need expert knowledge for classification purpose in contrast to fuzzy-based approaches (Moravej et al. 2015). As advantages, the number of support vectors increases when the size of the training set increases.

\subsection{Multilayer Perceptron Classifier}

Multilayer perceptron (MLP) is a feed-forward NN typically consisting of an input layer, one or several hidden layers, and one output layer. MLP networks are very useful for the classification of input signals when these cannot be defined mathematically. The algorithm can be trained to learn the existing relationships between input and output data (including nonlinear). Once they have been learned by example, they can be generalized. However, MLP has difficulties in determining a proper architecture, such as the number of hidden layers and nodes. Training MLPs is time-consuming without guaranteeing the global minimum (Lieberman et al. 2011).

Artificial neural networks for the classification of power system disturbances and faults have been extensively studied; some of the previous work can be found in (Rodriguez et al. 2014) and (Monedero et al. 2007). In (Rodriguez et al. 2014), a new dual neural network-based methodology to detect and classify single and combined PQ disturbances is proposed. A neural network is used to classify sags, swells, spikes, notching, flickers, harmonics, and inter-harmonics. In (Monedero et al. 2007), the researchers presented a system based on neural networks for the classification of electrical disturbances in real time. 


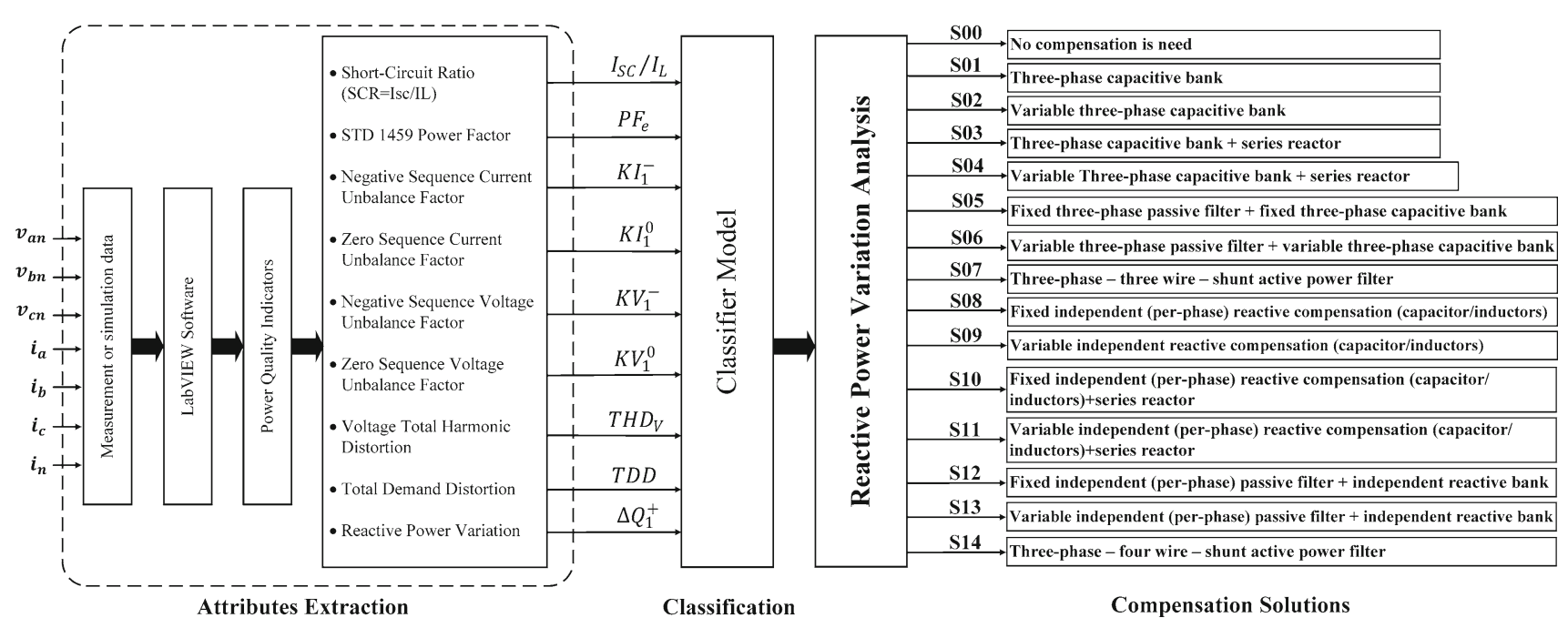

Fig. 1 Schematic diagram of the proposed methodology

All in all, many challenges in power systems have already been solved using different pattern recognition algorithms. However, power factor improvement, reactive and harmonic compensation are still open challenges.

\section{Proposed Methodology for Evaluating Pattern Recognition Algorithm for Power Factor Improvement}

Considering each previously mentioned PRA, one at a time, this section proposes a methodology to develop eleven different classifier models, which will be compared to each other, in order to determine the most suitable classifier for the development of an specialized system capable of pointing out the most proper power factor compensator for a certain installation. The proposed work consists of two stages: training and testing. In the first stage, we show the generation of an historical database with a known set of input-labeled and known responses (labels or classes) used in off-line training and development of classifier models. In the test stage, the generated classifying models are evaluated using a test database by means of computational simulation. Finally, the models are tested using real data samples, in order to evaluate the PRA algorithms with real cases.

Figure 1 shows four blocks that are used in the proposed methodology to evaluate each classifier model (DT, $K-\mathrm{NN}$, SVM, and MLP).

\subsection{Attributes Extraction}

Distribution power systems comprise numerous classes of disturbances, such as voltage or current unbalance, voltage or current distortion, and low power factor, which will be considered as relevant attributes for designing a suitable com- pensator, as shown in Fig. 1. Hence, the applied attributes are described as follows:

- Short-circuit ratio $\left(I_{\mathrm{SC}} / I_{\mathrm{L}}\right)$ : given by the ratio of shortcircuit current $\left(I_{\mathrm{SC}}\right)$ and the fundamental component of the maximum load current $\left(I_{\mathrm{L}}\right)$ (average value of maximum demand over the previous 12 months) (IEEE 2014);

- Voltage total harmonic distortion $\left(\mathrm{THD}_{V}\right)$ : This attribute is used in order to quantify and evaluate the harmonic distortion in voltage waveforms;

- Total demand distortion (TDD): given by the harmonic distortion of current expressed as a percentage of the maximum load current. Limits on current distortion depend on the power of the load compared with the power of the supply system at the PCC. The short-circuit currents at the PCC should be calculated to apply IEEE criteria of TDD at PCC (IEEE 2014);

- Equivalent Power factor $\left(\mathrm{PF}_{\mathrm{e}}\right)$ : It represents the fraction of the power supplied by a utility that is used to perform a useful work. It is frequently used by the utilities to penalize the customer. In this work, the power factor is calculated according to STD 1459 (IEEE 2010);

- Voltage unbalance factors $\left(\mathrm{KV}_{1}^{-}\right)$or $\left(\mathrm{KV}_{1}^{0}\right)$ : indicate unequal voltage magnitudes at the fundamental frequency, fundamental phase angle deviation, and imbalanced levels of harmonic distortion among phases. These are other important attributes used in this work to indicate the uneven distribution of load through a three-phase power system. These terms consider the RMS value of the fundamental positive, negative, or zero sequence and can be obtained using Fortescue's symmetrical components;

- Current unbalance factor $\left(\mathrm{KI}_{1}^{-}\right)$or $\left(\mathrm{KI}_{1}^{0}\right)$ : may be caused by large or unequal distribution of single phase load, 
phase to phase loads or unbalanced three-phase loads. They are used to evaluate the load unbalance condition and can also be calculated based on symmetrical components;

- Positive-sequence reactive power variation $\left(\Delta Q_{1}^{+}\right)$: This attribute will be used in order to analyze the reactive power over the time.

\subsubsection{Classification: Standards and Adopted Limits}

According to Fig. 1, this block is responsible for classifying the input data, by means of the chosen and trained PRA, Fig. 1, and the calculated attributes, before the final classification in terms of the reactive power's variability. At this point, the specialist's knowledge is already considered in the classifier and there is no need for any adaptation of the classifier rules.

\subsubsection{Reactive Power Variation}

In order to evaluate the reactive power variations over time, this step is responsible to check whether the variations are above or below $\pm 30 \%$ average over $24 \mathrm{~h}$. Thus, this stage provides information regarding the required compensator. If the reactive power variation is above $30 \%$, the compensator should be "switched" (like thyristor-switched capacitors) to provide exactly the right amount of reactive power at the right time according to load requirements. Otherwise, the compensator should provide a constant value of reactive power (like fixed capacitor banks).

\subsubsection{Compensation Solutions}

The chosen compensator or solution have a vital role to play in maintaining supply voltages within the specified limits, improving the power factor, reducing the harmonic distortion, and balancing the load currents. Thus, the last step in Fig. 1 presents a list of possible solutions according to the "classification block" and the "reactive power variation analysis." Notice that the solution includes lowfrequency compensators ("Fixed" and/or "Switched") and high-frequency compensators (like active power filters).

For a particular set of input data, it is clear that the proposed solution partly depends on the available attributes and limits during the training and classification, but mostly on the chosen PRA for the classifier construction, as discussed in next sections.

\subsection{Training Stage}

This stage is responsible for the training of the chosen PRA based on the applicable attributes, specialized knowledge, and a set of historical simulation and measurement data, in

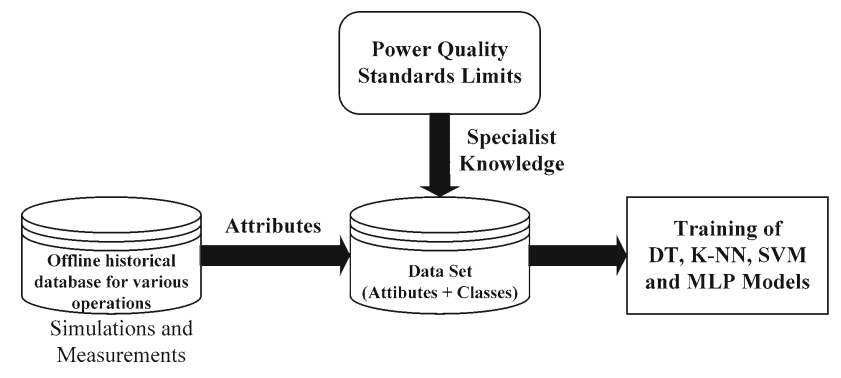

Fig. 2 Flowchart of the training stage

such a way that the resulting decision rules can classify new samples of a test data, automatically, by simply using the test data attributes, Fig. 2.

It is worth mentioning that the decision rules for each PRA are dynamically defined during this stage. Taking, for example, the DT method, the training process results in an automatic self-structured DT.

The training data set was developed considering several simulations of different compensation examples, which have been generated and characterized by a human specialist, in order to perform a supervised learning by means of supplying a known set of input-labeled training data (power quality indicators) and known responses (compensation cases). The specialist, taking into account the $\mathrm{PQ}$ standards limits: equivalent power factor $\left(\mathrm{PF}_{\mathrm{e}}>0.92\right)$, the unbalance factors for the voltages $\left(\mathrm{KV}_{1}^{-}\right.$or $\left.\mathrm{KV}_{1}^{0}<2 \%\right)$, the unbalance factors for the currents $\left(\mathrm{KI}_{1}^{-}\right.$or $\left.\mathrm{KI}_{1}^{0}<20 \%\right)$, total demand distortion, voltage total harmonic distortion $\left(\mathrm{THD}_{V}<5 \%\right.$ ), evaluates extensively the successful simulations and measurement cases. All simulations were performed at a voltage level between $120 \mathrm{~V}$ and $69 \mathrm{kV}$. For each type of disturbing load, a subset of attributes was generated (1355154 samples) using PSCAD environment. The PSCAD software allows the use of various control tools to perform parameter modification simulation. In addition, the PSCAD allows to perform the simulation of large power systems. Besides, several real measurements, evaluated by the specialist, were also added to this database. The classifier models were built and trained using the tool "Classification Learner" included in the MATLAB software (Statistics and Machine Learning Toolbox 2017). The Classification Learner App is an application that can be used to train models for classification of data using supervised machine learning methods. By using Classification Learner, it is possible to explore data, select features, specify validation schemes, train models, and assess results. The prediction models developed in this study have been trained, tested, and evaluated using a fivefold cross-validation technique.

The decision tree models were developed using the Classification and Regression Tree (CART) algorithm (Breiman et al. 1984). Hence, the leaves represent the classification results (compensation solutions), and the nodes represent 
attributes. A CART algorithm searches all variables for all possible values, in order to find the best split. The split criterion used is Gini's diversity index. Three decision tree models have been developed using different depths (4,20, and 100), which are the highest number of levels used in the decision tree.

In this work, three $K$-NN models have been developed using different $K$ values (1,3, and 10), which are the number of neighbors. In addition, the Euclidean distance metric was used to measure the distance between patterns in this study.

The prediction SVM model developed in this study has been developed using the linear kernel function and "oneversus-one" multiclass strategy.

For MLP training, we used as function fitting a two-layer feed-forward network, with a sigmoid transfer function in the hidden layer and a linear transfer function in the output layer. The number of hidden neurons for each MLP model was 10, 20, 40, and 50, respectively. In addition, for the MLP training, we used up to 1000 epochs and the backpropagation learning algorithm.

Regarding the limits used by the specialist to be applied for the chosen attributes, some come directly from particular standards, while others do not have specific boundaries in the literature, and the specialist would usually define them in each case. In such cases, during the training stage, the classifier can also set the lacking limits automatically, in order to achieve the compensation results according to the input training data.

Regardless of the source, high harmonic voltage levels and harmonic load currents will lead to operational problems in the electric power distribution system. These problems include equipment-heating, over voltage, and load disruption and have been discussed in IEEE 519 (IEEE 2014). This standard provides recommended practices for the harmonic evaluation of electrical power systems and is widely accepted by industry and utilities. European standard (EN 501602004 ) sets the boundaries for harmonic voltages. IEEE 519 has $5 \%$ to the voltage total harmonic distortion limit and $3 \%$ to individual harmonic voltage. In this work, the authors adopted a conservative limit of $5 \%$ for total harmonic distortion $\left(\mathrm{THD}_{V}\right)$ to avoid excessive voltage distortion on capacitor banks.

Concerning the limits for harmonic current, it is observed that both IEC (IEC 2008, 1996) and IEEE (IEEE 2014) deal with the limitation of harmonic currents in low voltage (LV). However, IEEE 519 considers the short-circuit ratio $\left(I_{\mathrm{SC}} / I_{\mathrm{L}}\right)$ to take into account the size of the load in regard to the system, when setting the limits on the harmonic current emission. In this way, the current harmonic distortion will be evaluated according to IEEE 519 by means of TDD.

The power factor is considered by the utility for billing purposes and may lead to monthly charges for the consumers. In this work, the adopted power factor limit was 0.92 , accord-
Table 1 Accuracy and speed of classification methods for the training set

\begin{tabular}{lll}
\hline Classification method & Training time (s) & Accuracy (\%) \\
\hline DT (depth $=4)$ & 14 & $71.70 \pm 1.53$ \\
DT (depth $=20)$ & 11 & $100 \pm 0.01$ \\
DT (depth $=100)$ & 12 & $100 \pm 0.01$ \\
$K$-NN $(K=1)$ & N/A & $100 \pm 0.00$ \\
$K$-NN $(K=3)$ & N/A & $100 \pm 0.01$ \\
$K$-NN $(K=10)$ & N/A & $100 \pm 0.01$ \\
Linear SVM & 4987 & $100 \pm 0.01$ \\
MLP $(10$ neurons) & 2224 & $99.90 \pm 0.08$ \\
MLP $(20$ neurons $)$ & 2648 & $99.80 \pm 0.08$ \\
MLP (40 neurons) & 3364 & $99.80 \pm 0.08$ \\
MLP (50 neurons) & 3091 & $99.89 \pm 0.07$ \\
\hline
\end{tabular}

ing to the Brazilian Electrical Regulatory Agency (ANEEL 2017).

Regarding the voltage unbalance, the ANSI C84.1-2006 standard recommends that electrical supply systems should operate with a maximum voltage unbalance limit of $3 \%$ (ANSI 2006). The IEC standard recommends that the maximum voltage unbalance of electrical supply systems should be limited to $2 \%$ (IEC 2008, 1996). In "IEEE Recommended Practice for Electric Power Distribution for Industrial Plants" (IEEE 1993), and ANSI/IEEE Std. 241-1990, "IEEE Recommended Practice for Electric Power Systems in Commercial Buildings" (IEEE 1990), both the Red Book and the Gray Book indicate that some electronic equipment (like computers) may experience problems if the voltage unbalance is more than 2-2.5\%. Therefore, based on (IEEE 1993, 1990; NEMA 1993), the authors adopted $2 \%$ as a limit for the attribute voltage unbalance.

To evaluate the unbalanced load, the authors propose the current unbalance factor. This attribute is not defined in standards. However, the National Equipment Manufacturers Association (NEMA) (NEMA 1993) states that $1 \%$ of voltage unbalance can create $6-10 \%$ current unbalance. Thus, by limiting the voltage unbalance to $2 \%$, the authors propose the limit of $20 \%$ as current unbalance factor.

The accuracy and speed of the training process are given in Table 1, for each PRA. It becomes evident that DT, $K$ $\mathrm{NN}$, and SVM can correctly classify the possible cases using the training data. However, it is important to test the models for different data sets in order to evaluate the performance for imbalance data. The problem with the imbalance class has been recognized as a crucial problem in machine learning and pattern recognition areas. Significant differences in class prior probabilities may produce an important deterioration of the classification systems' performance. A two-class data set is imbalanced when one of the classes (the minority one) is heavily underrepresented in comparison with another 
Table 2 Confusion matrix (Sokolova and Lapalme 2009)

\begin{tabular}{lll}
\hline Data class & Classified as positives & Classified as negatives \\
\hline Real positives & TP & FN \\
Real negatives & FP & TN \\
\hline
\end{tabular}

class (the majority one). This issue is particularly important in real-world applications where it is costly to misclassify examples from the minority class, such as diagnosis of rare diseases, detection of fraudulent telephone calls, and filtering tasks. In the experimental results section, this problem will be discussed considering real-world data. Note that SVM and MLP need a large training time increasing the computation cost.

After the training process, each resulting decision-maker was ready to be used as a tool for identifying the compensation solutions for power factor improvement.

\section{Performance Indices for Classifiers Evaluation}

Designing a classifier is mathematically equivalent to finding a mapping function between the input space and the attribute space, followed by applying decision rules that then map the attribute space into the decision space. In this way, the four pattern recognition classifiers presented in Sect. 2 (DT, $K$ $\mathrm{NN}, \mathrm{SVM}$, and MLP) were used in the classification task, and their results compared in order to find the best classifier in the PQ problem studied here.

Regarding the performance evaluation, many metrics have been employed to assess the classification effectiveness. All of them are based on the confusion matrix as shown in Table 2. The correctness of a classification can be evaluated by computing the number of correctly recognized class examples ( $T P$ - true positives), the number of correctly recognized examples that do not belong to the class ( $T N-$ true negatives), and examples that either were incorrectly assigned to the class ( $F P$-false positives) or were not recognized as class examples ( $F N$-false negatives) (Sokolova and Lapalme 2009).

Accordingly, from Table 2, the overall effectiveness of a classifier can be measured as:

accuracy $=\frac{\mathrm{TP}+\mathrm{TN}}{\mathrm{TP}+\mathrm{FP}+\mathrm{FN}+\mathrm{TN}}$

In order to evaluate how effectively a classifier identifies negative labels, we consider:

specificity $=\frac{\mathrm{TN}}{\mathrm{FP}+\mathrm{TN}}$

The corresponding effectiveness of a classifier to identify positive labels is as follows:

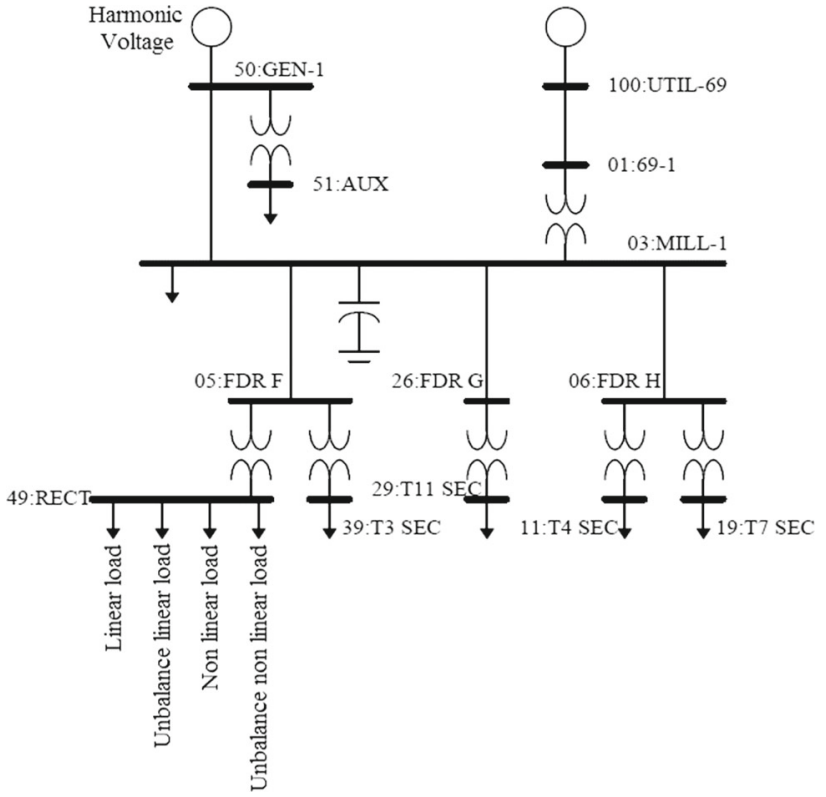

Fig. 3 Modified IEEE 13-bus balanced industrial system test feeder

recall $=\frac{\mathrm{TP}}{\mathrm{TP}+\mathrm{FN}}$

It is important to find the performance measurement that has not been influenced by the imbalanced data. Thus, $G$ mean is a measure of the classifier's ability to balance sensitivity and specificity and can be defined as follows:

$G$-mean $=\sqrt{\text { recall } \cdot \text { specificity }}$

Area under the curve (AUC) is the area below the receiver operating characteristic (ROC) (Sokolova and Lapalme 2009) curve that depicts the performance of a classifier. It has been proved to be a reliable performance measure for class imbalance problem. It can measure the classifier's ability to avoid false classification and can be expressed by:

$\mathrm{AUC}=\frac{1}{2}\left(\frac{\mathrm{TP}}{\mathrm{TP}+\mathrm{FN}}+\frac{\mathrm{TN}}{\mathrm{TN}+\mathrm{FP}}\right)$

\section{Computational Results}

At this point, the specialist's knowledge is already considered in the classifier and there is no need for any adaptation of the classifier rules. After training the classifier models, in order to verify the generalization capacity of the developed classifiers, a new test database was developed using the IEEE 13-Bus Balanced Industrial System, shown in Fig. 3.

The IEEE 13-bus system was used to create a validation balanced data set $(111,055$ samples $)$ used to compare the 


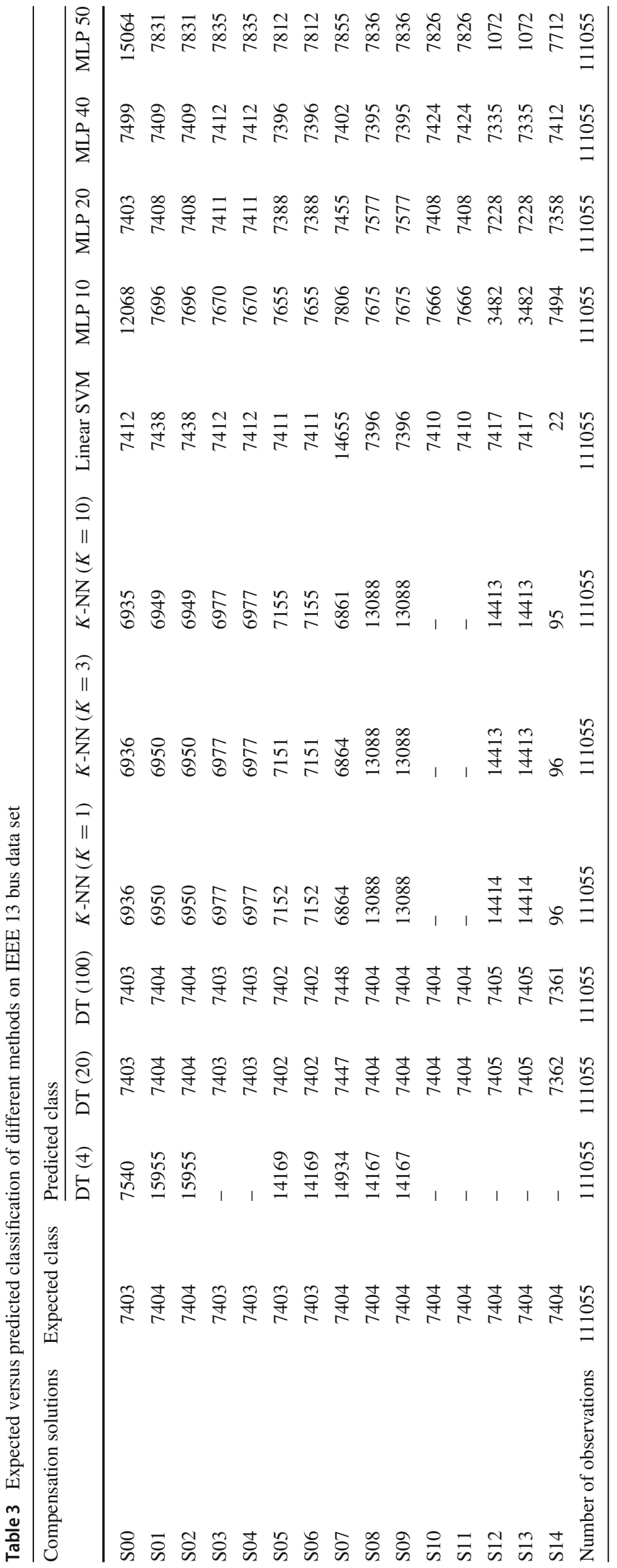


Table 4 Evaluation of different classification methods on IEEE 13 bus data set

\begin{tabular}{|c|c|c|c|c|}
\hline \multirow[t]{2}{*}{ Classification methods } & \multicolumn{4}{|c|}{ Performance metrics (\%) } \\
\hline & Accuracy & Specificity & $G$-mean & AUC \\
\hline DT $($ depth = 4) & $55.93 \pm 2.39$ & 51.07 & 71.47 & 75.54 \\
\hline DT $($ depth $=20)$ & $99.93 \pm 0.03$ & 99.92 & 99.96 & 99.96 \\
\hline DT $($ depth = 100) & $99.93 \pm 0.03$ & 99.92 & 99.96 & 99.96 \\
\hline$K$-NN $(K=1)$ & $76.79 \pm 1.78$ & 74.24 & 86.13 & 87.08 \\
\hline$K$-NN $(K=3)$ & $76.80 \pm 1.83$ & 74.25 & 86.13 & 87.08 \\
\hline$K$-NN $(K=10)$ & $76.79 \pm 1.63$ & 74.25 & 86.13 & 87.08 \\
\hline Linear SVM & $88.48 \pm 0.59$ & 87.21 & 93.39 & 93.61 \\
\hline MLP (10 neurons) & $93.96 \pm 0.41$ & 93.30 & 96.59 & 96.65 \\
\hline MLP (20 neurons) & $99.57 \pm 0.08$ & 99.53 & 99.76 & 99.76 \\
\hline MLP (40 neurons) & $99.77 \pm 0.04$ & 99.75 & 99.87 & 99.87 \\
\hline MLP (50 neurons) & $90.76 \pm 0.47$ & 89.74 & 94.73 & 94.87 \\
\hline
\end{tabular}

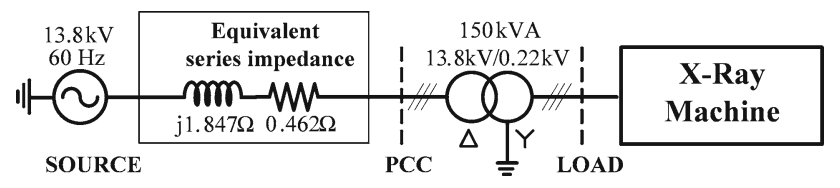

Fig. 4 Single-line diagram of the power system

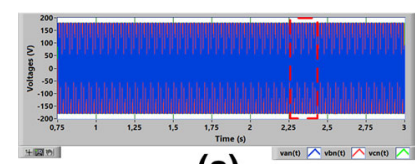

(a)

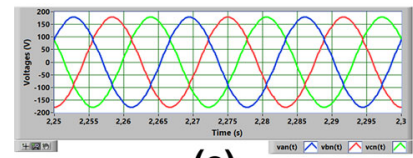

(c)

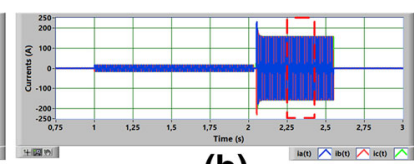

(b)

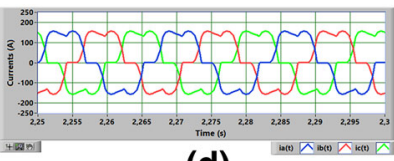

(d)
Fig. 5 Three-phase X-ray machine voltages and currents

performance of trained classifiers, by means of a supervised test, and decide which one to take, thus allowing the classifier's performance evaluation by means of simulation results. This system consists of 13 buses, $69 \mathrm{kV}$ power supply from the utility, and seven transformers. A $6 \mathrm{MVAr}$ rated power factor capacitor is connected at bus 3 in medium voltage $(13.8 \mathrm{kV})$. Specific issues related to cables, transformers, and load characteristics are described by the data in (Abu-Hashim et al. 1999). In order to take into account the load variation effects and the possible disturbances (harmonic current, harmonic voltage, current unbalance, voltage unbalance, a low power factor, and their combinations), a group of linear and nonlinear loads has been inserted at 49:RECT bus. Besides, in order to simulate the voltage distortions from the utility perspective, a programmable voltage source was inserted at 50:GEN-1 bus. In this way, it is possible to reproduce different combinations of disturbances to be analyzed by the developed classifier model. In addition, with the combina- tion of the different types of loads, it is possible to generate samples for all classes (S00 to S14), since the objective is to analyze the capacity of the classifiers in separating the samples from the respective classes.

By applying the proposed methodology, the voltages and currents were measured at 49:RECT bus with a sampling frequency of $12.6 \mathrm{kHz}$. According to Fig. 1, the required voltage, current, and power attributes were calculated and stored (attributes extraction).

Table 3 shows the classification results performed by each technique chosen in this work. The compensation solutions column represents the possible classes. The expected class column represents the known responses defined by power engineer (expert) during the creation of the testing database. In this stage, the attributes described in Sect. 3.1 are provided to the classifier models developed in the training phase. Thus, the classifier under analysis performs the separation of the respective classes. The output of each classifier is then compared to the known response. It can be seen from Table 3 that the DT with depth (4) has difficulties in identifying the cases S01, S02, S05, S06, S07, S08, and S09. In addition, this classifier model was unable to identify the samples present in classes S03, S04, S10, S11, S12, S13, and S14. As the depth of the decision tree is increased, it is possible to observe the classifier ability to separate classes correctly, according to results obtained through the use of the DT with depth (100). In the case of the $K-\mathrm{NN}$, it is difficult for the classifier to identify classes S10 and S11, even with the increase in the value of $K$. In the case of the linear SVM technique, an improvement in the identification of the classes S10 and S11 is verified when compared to the $K$-NN technique. However, this classifier cannot correctly identify the samples present in class S14, that is, there is a difficulty in identifying the solution "Three-phase - four wire shunt active power filter." In the case of the MLP technique with ten neurons, it is observed that the classifier identified 


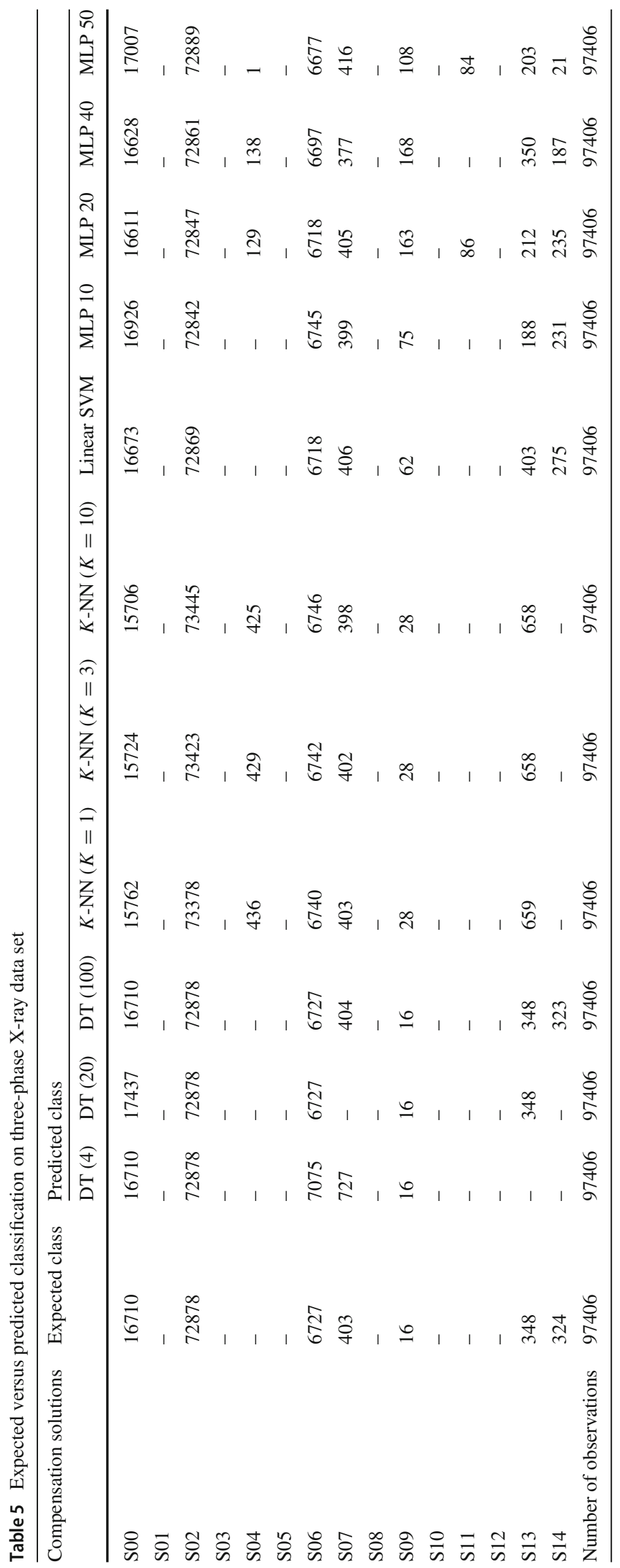


Table 6 Evaluation of different classification methods on X-ray data set

\begin{tabular}{|c|c|c|c|c|}
\hline \multirow[t]{2}{*}{ Classification methods } & \multicolumn{4}{|c|}{ Performance metrics (\%) } \\
\hline & Accuracy & Specificity & $G$-mean & AUC \\
\hline DT $($ depth $=4)$ & $99.31 \pm 0.62$ & 97.26 & 98.62 & 98.64 \\
\hline DT $($ depth $=20)$ & $100 \pm 0.01$ & 100 & 100 & 100 \\
\hline DT $($ depth $=100)$ & $100 \pm 0.00$ & 100 & 100 & 100 \\
\hline$K$-NN $(K=1)$ & $98.94 \pm 0.05$ & 99.91 & 97.05 & 97.09 \\
\hline$K$-NN $(K=3)$ & $98.90 \pm 0.04$ & 99.91 & 96.93 & 96.97 \\
\hline$K$-NN $(K=10)$ & $98.88 \pm 0.05$ & 99.91 & 96.86 & 96.91 \\
\hline Linear SVM & $99.95 \pm 0.03$ & 99.99 & 99.87 & 99.87 \\
\hline MLP (10 neurons) & $99.78 \pm 0.06$ & 99.73 & 99.87 & 99.87 \\
\hline MLP (20 neurons) & $99.73 \pm 0.05$ & 99.85 & 99.52 & 99.52 \\
\hline MLP (40 neurons) & $99.80 \pm 0.03$ & 99.93 & 99.54 & 99.55 \\
\hline MLP (50 neurons) & $99.42 \pm 0.01$ & 99.41 & 99.43 & 99.43 \\
\hline
\end{tabular}

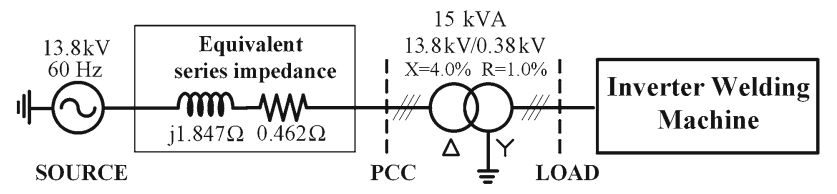

Fig. 6 Single-line diagram of the power system

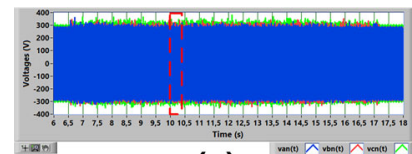

(a)

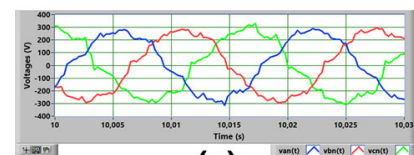

(c)

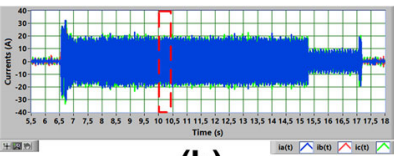

(b)

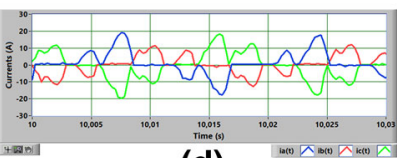

(d)
Fig. 7 Inverter welding machine voltages and currents

12068 samples for class 0, that is, 4665 samples classified incorrectly. In addition, a difficulty of this classifier in correctly identifying the samples of classes S12 and S13 is observed. With the increase in the number of neurons for the MLP, it is possible to observe the improvement in the capacity of the classifier model to identify classes S0, S12, and S13.

The objective of any classifier is the maximization of accuracy, specificity, $G$-mean, and AUC. In this way, these criteria will be applied for assessing the algorithms' performance. Table 4 shows the results of several classification methods when the IEEE 13-bus data set is considered. We can observe that DT with depth 4 has the worst accuracy $(55.93 \%)$ and AUC criterion (75.54\%). However, when the tree depth is increased to 20 , we observe that the DT classifier obtains the best performance compared to other methods.

\section{Experimental Results}

Looking for the evaluation of each classifier performance in practical applications, three actual test sets composed by three disturbing loads, which result in imbalanced data sets, were analyzed by means of supervised classification. The measurements have been performed in different installations in Brazil, which claim lack of power quality in their respective facilities.

As shown in Fig. 1, the voltages and currents are measured by means of a data acquisition system and used to calculate the attributes described in Section 3.1. Following, these attributes are classified according to the resulting classifier models.

\subsection{Three-Phase X-Ray Machine}

The first measurement is related to an X-ray machine. This kind of load is considered as disturbing due to its operation mode. During the time of radiography, the required electrical power is high and can produce voltage sags or even damage the operation of other sensitive equipment connected to the circuit. Figure 4 shows the single-line diagram of the power system under analysis. The measurements were performed on the low-voltage side (220V).

Figure 5 shows the load instantaneous currents, measured in X-ray equipment $220 \mathrm{~V}, 60 \mathrm{~Hz}$, during the execution of radiography (Moreira et al. 2015). Note from Fig. 5 that the current has amplitude variant over time, which may result in different compensation solutions over time.

Table 5 presents the known responses (expected class) versus predictions made by the trained models. Note that the pattern recognition decision-tree algorithm $($ depth $=4)$ fails to identify S13 and S14 solutions.

From Table 6, we observe that all classifiers reach high accuracy. Observe that the pattern recognition decision-tree 


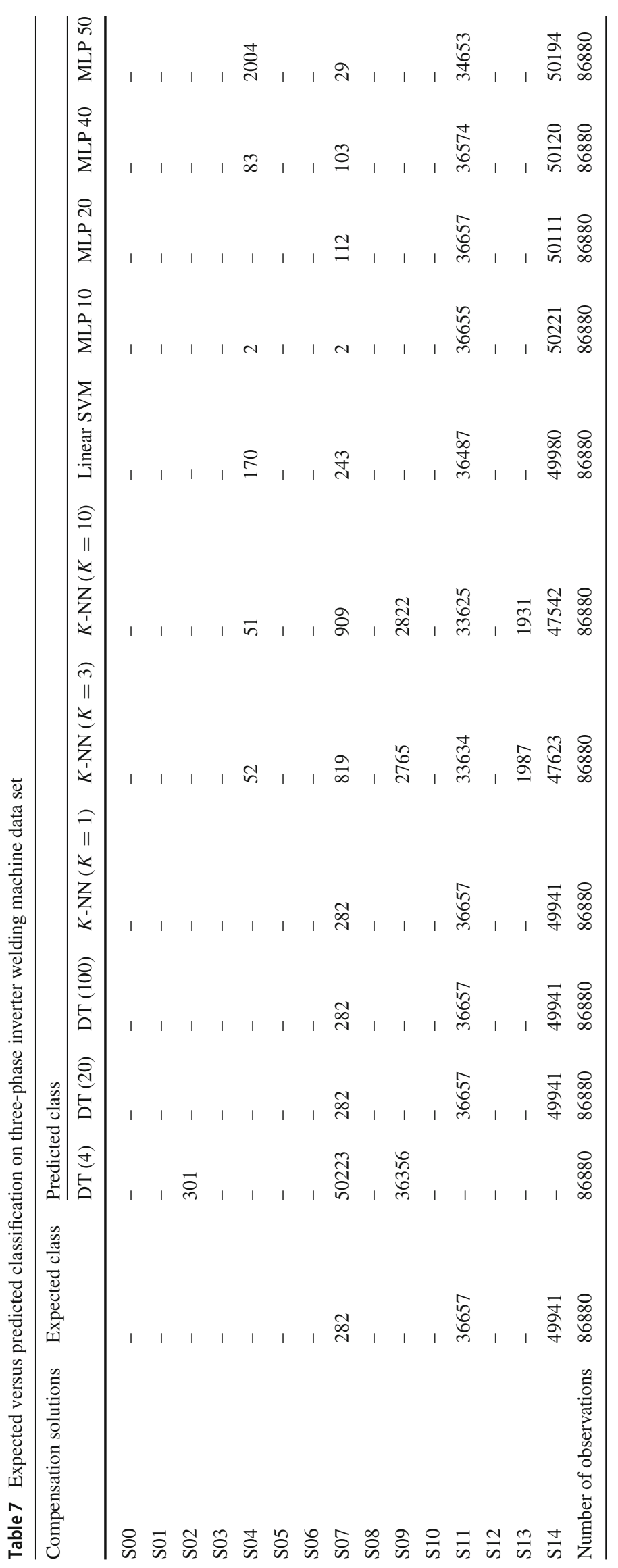


Table 8 Evaluation of different classification methods on three-phase inverter welding machine data set

\begin{tabular}{lllll}
\hline Classification methods & \multicolumn{2}{l}{ Performance metrics $(\%)$} & $G$-mean & AUC \\
\cline { 2 - 4 } & Accuracy & Specificity & 0.00 & 50.00 \\
\hline DT (depth $=4)$ & $0.32 \pm 3.44$ & 0.00 & 100 & 100 \\
DT (depth $=20)$ & $100 \pm 0.00$ & 100 & 100 & 100 \\
DT (depth $=100)$ & $100 \pm 0.01$ & 100 & 51.52 & 60.97 \\
$K$-NN $(K=1)$ & $93.37 \pm 0.07$ & 93.58 & 50.88 & 60.63 \\
$K$-NN $(K=3)$ & $93.38 \pm 0.11$ & 93.60 & 50.20 & 60.22 \\
$K$-NN $(K=10)$ & $93.27 \pm 0.12$ & 93.49 & 92.74 & 92.99 \\
Linear SVM & $99.76 \pm 0.03$ & 99.80 & 8.42 & 50.35 \\
MLP $(10$ neurons) & $99.68 \pm 0.02$ & 100 & 51.21 & 63.10 \\
MLP (20 neurons) & $99.72 \pm 0.09$ & 99.96 & 60.41 & 68.21 \\
MLP (40 neurons) & $99.70 \pm 0.10$ & 99.90 & 23.54 & 51.67 \\
MLP (50 neurons) & $97.37 \pm 0.14$ & 97.67 &
\end{tabular}

algorithm (depth $=20$ and 100) has the best results for all and $\Delta Q_{1}^{+}$violations. As we can see, increasing the number of neurons does not result in a better accuracy or AUC. metrics resulting in compensation solution $\mathrm{S} 02$, due to $\mathrm{PF}_{\mathrm{e}}$

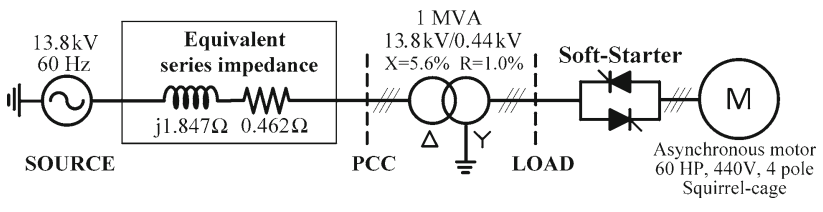

Fig. 8 Single-line diagram of the power system

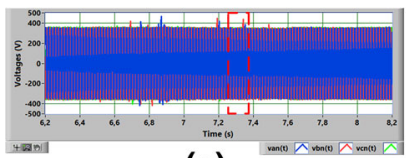

(a)

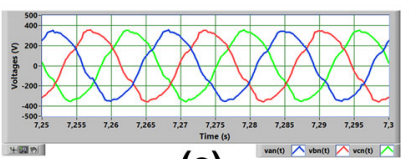

(c)

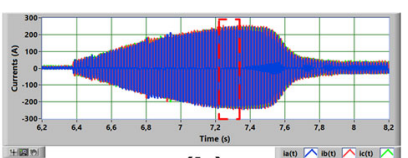

(b)

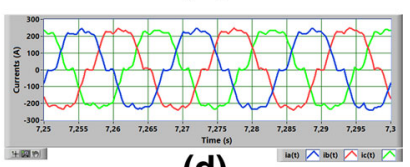

(d) voltages and currents are distorted, while the currents are also unbalanced.

For this data set, the known responses versus the predicted classification are shown in Table 7. In this case, the compensation solution suggested is $\mathrm{S} 14$ due to higher incidence of $\mathrm{PF}_{\mathrm{e}}, \mathrm{KI}_{1}^{-}, \mathrm{THD}_{I}, \mathrm{THD}_{V}$, and $\Delta Q_{1}^{+}$violations. Note that $\mathrm{DT}$ $($ depth $=4)$ has misclassified 36356 samples as S09.

Table 8 summarizes the metrics for all classifiers when applied to the welding machine data set. Again, one can observe that DT (depth $=4$ ) has the worst accuracy and AUC. This means that the solution provided by this PRA, in this case, will be S07 with 50,223 samples misclassified, Table 7. In this instance, we can perceive the importance of all different metrics. The MLP (ten neurons) has a high accuracy (99.68\%), however with a low AUC (50.35). This occurs because this PRA only classified two samples as S07 instead of 282 samples. The $K-\mathrm{NN}$ model has misclassified some samples as S09, resulting in a low AUC. In contrast, the decision tree (depth 20 and 100) has a 100\% result for all metrics, in other words, all attributes were correctly classified.
Fig. 9 Soft-starter voltages and currents

\subsection{Soft Starter}

Soft starters are generally employed in large capacity induction motors to limit the initial current and to achieve smooth starting. However, they cause severe pollution on the supply such as harmonic distortion.

Figure 8 shows the single-line diagram of the power system under analysis for the induction motor $60 \mathrm{HP}, 440 \mathrm{~V}$, $60 \mathrm{~Hz}$ with soft starter. The measurements were performed in the low-voltage side (440 V), as can be seen in Fig. 8.

Figure 9 shows the voltages and currents' waveforms for the induction motor $60 \mathrm{HP}, 440 \mathrm{~V}, 60 \mathrm{~Hz}$ with soft starter. Note that voltages and currents are distorted and variant over time.

For this data set, the known responses are S05 (140122 samples), S07 (64500 samples), and S01 (466 samples) with 


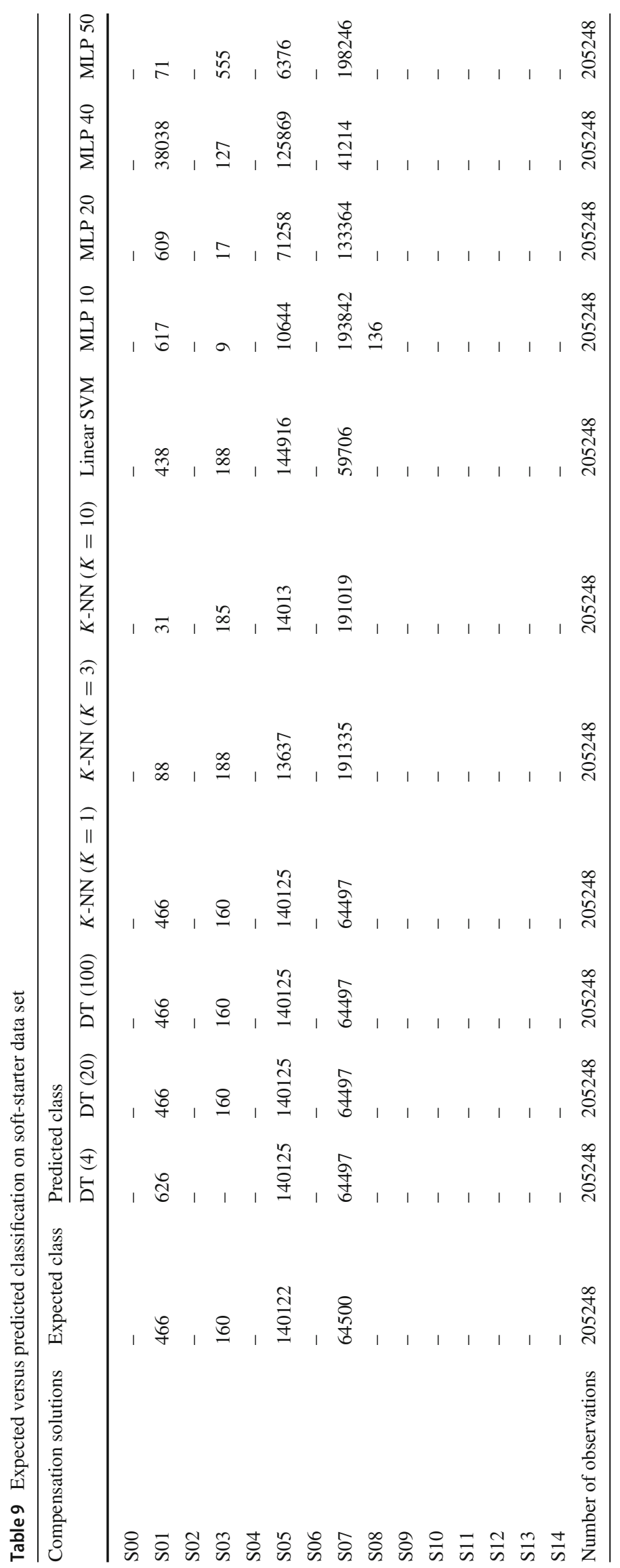


Table 10 Evaluation of different classification methods on soft-starter data set

\begin{tabular}{|c|c|c|c|c|}
\hline \multirow[t]{2}{*}{ Classification methods } & \multicolumn{4}{|c|}{ Performance metrics (\%) } \\
\hline & Accuracy & Specificity & $G$-mean & AUC \\
\hline DT $($ depth = 4) & $99.92 \pm 0.02$ & 99.92 & 99.96 & 99.96 \\
\hline DT $($ depth $=20)$ & $100 \pm 0.00$ & 100 & 100 & 100 \\
\hline DT $($ depth = 100) & $100 \pm 0.01$ & 100 & 100 & 100 \\
\hline$K-\mathrm{NN}(K=1)$ & $99.76 \pm 0.02$ & 99.97 & 24.51 & 52.99 \\
\hline$K-\mathrm{NN}(K=3)$ & $99.76 \pm 0.03$ & 99.97 & 24.51 & 52.99 \\
\hline$K-\mathrm{NN}(K=10)$ & $99.79 \pm 0.02$ & 100 & 25.79 & 53.33 \\
\hline Linear SVM & $99.99 \pm 0.01$ & 100 & 96.95 & 97.00 \\
\hline MLP (10 neurons) & $99.86 \pm 0.05$ & 99.86 & 99.93 & 99.93 \\
\hline MLP (20 neurons) & $99.93 \pm 0.04$ & 99.93 & 99.97 & 99.97 \\
\hline MLP (40 neurons) & $81.69 \pm 0.05$ & 81.65 & 90.36 & 90.83 \\
\hline MLP (50 neurons) & $99.81 \pm 0.06$ & 100 & 39.03 & 57.62 \\
\hline
\end{tabular}

$\mathrm{PF}_{\mathrm{e}}, \mathrm{THD}_{I}$, and $\mathrm{THD}_{V}$ violations, Table 9. Note that MLP (ten neurons) has misclassified 136 samples as S08.

Table 10 shows the classifiers metrics for this data set. It is interesting to observe that the $K$-NN accuracy is improved for this kind of load, as well as the linear SVM classifier. However, the AUC for $K-\mathrm{NN}$ is around $53 \%$ and the $G$ mean metric about $24 \%$, meaning that $K-\mathrm{NN}$ ( $K=1,3$, and 10), MLP (10, 20, and 50 neurons) will result in S07 as first compensation solution (higher incidence) instead of S05. On the other hand, the decision-tree algorithm with depth 4 has reached $99.92 \%$ of accuracy and $99.96 \%$ of AUC. However, by increasing the tree depth, we can observe a performance improvement, so that the AUC reaches $100 \%$ for depths 20 and 100 .

\section{Conclusion}

In this paper, we compared several pattern recognition algorithms in order to automate the usual human decision-making process on power factor improvement in distribution systems. We have employed international standards as IEEE, IEC, ANSI, and NEMA to assess the different power quality indicators in order to analyze the power system's behavior. The decision-making process about the reactive, unbalanced, and harmonic compensation was performed using different algorithms, and our results indicate that an automatic self-structured decision-tree algorithm, with depth 20 , is highly effective and reliable to classify the chosen attributes. The developed tool may also decrease the need for power system or power quality experts to perform the compensation analysis insofar as our proposed framework indicates (quasi-autonomously) the most suitable compensation solution, directly obtained from the input data.
Acknowledgements The authors gratefully acknowledge the contributions of São Paulo Research Foundation (FAPESP) under Grant 2016/08645-9 and by Finnish Academy and CNPq/Brazil (n.490235/20 12-3) as part of the joint project SUSTAIN, by Strategic Research Council/Aka BC-DC project (n.292854) for their financial support toward the development of this research.

\section{References}

Abu-Hashim, R., et al. (1999). Test systems for harmonics modelling and simulations. IEEE Transactions on Power Delivery, 14(2), 579-587.

Andreoli, A. L., Coury, D. V., Oleskovicz, M., \& Serni, P. A. (2013). Artificial neural network model of discharge lamps in the power quality context. Journal of Control, Automation and Electrical Systems, 24(3), 272-285.

ANEEL. (2017). Procedimentos de Distribuição de Energia Elétrica no Sistema Elétrico Nacional-PRODIST, Módulo 8-Qualidade da Energia Elétrica. Agência Nacional de Energia Elétrica - ANEEL, revisão 8, vigente a partir de January 01, 2017.

ANSI. (2006). Electric power systems and equipment—voltage ratings (60 Hertz). ANSI Standard C84.1-2011 (Revision of ANSI C84.12006).

Axelberg, P. G. V., Gu, I. Y. H., \& Bollen, M. H. J. (2007). Support vector machine for classification of voltage disturbances. IEEE Transactions on Power Delivery, 22(3), 1297-1303.

Barbosa, B. H. G., \& Ferreira, D. D. (2013). Classification of multiple and single power quality disturbances using a decision tree-based approach. Journal of Control, Automation and Electrical Systems, 24(5), 638-648.

Beyer, K., Goldstein J., Ramakrishnan, R., \& Shaft U. (1999). When is nearest neighbor meaningful? In Proceedings of the 7th international conference on database theory (pp. 217-235).

Biet, M. (2013). Rotor faults diagnosis using feature selection and nearest neighbors rule: application to a turbogenerator. IEEE Transactions on Industrial Electronics, 60(9), 4063-4073.

Bollen, M. H. J., \& Gu, I. Y. H. (2006). Signal processing of power quality disturbances (1st ed.). Hoboken: Wiley.

Breiman, L., Friedman, J., Stone, C. J., Olshen, R. A., \& Stone, C. I. (1984). Classification and regression trees (1st ed.). Boca Raton: Chapman and Hall/CRC. 
Cerqueira, A. S., Ferreira, D. D., Ribeiro, M. V., \& Duque, C. A. (2008). Power quality events recognition using a svm-based method. Elsevier Electric Power Systems Research, 78, 1546-1552.

Das, J. C. (2015). Power system harmonics and passive filter designs (1st ed.). Hoboken: Wiley.

EN 50160. (2004). Voltage characteristics of electricity supplied by public distribution systems. Standard EN 50160.

Galil, T. A., Kamel, M., Youssef, A. M., Saadany, E. F. E., \& Salama, M. M. A. (2004). Power quality disturbance classification using the inductive inference approach. IEEE Transactions on Power Delivery, 19(4), 1812-1818.

Gaouda, A. M., Kanoun, S. H., \& Salama, M. M. A. (2001). On-line disturbance classification using nearest neighbor rule. Elsevier Electric Power Systems Research, 57(1), 1-8.

Gaouda, A. M., Kanoun, S. H., Salama, M. M. A., \& Chikhani, A. Y. (2002). Pattern recognition applications for power system disturbance classification. IEEE Transactions on Power Delivery, 17(3), 677-683.

Hajian, M., \& Foroud, A. A. (2014). A new hybrid pattern recognition scheme for automatic discrimination of power quality disturbances. Elsevier Electric Power Systems Research, 51, 265-280.

IEC. (1996). Electromagnetic Compatibility (EMC)_LimitsAssessment of emission limits for distorting loads in MV and $H V$ power systems. International Electro Technical Commission Standard, 61000-3-6.

IEC. (2008). Electromagnetic Compatibility (EMC)-LimitsAssessment of emission limits for the connection of unbalanced installations to $M V, H V$ and EHV power systems. International Electro Technical Commission Standard, 61000-3-13.

IEEE. (1990). IEEE recommended practice for electric power systems in commercial buildings, ANSI/IEEE Std. 241-1990 (Gray Book).

IEEE. (1993). IEEE recommended practice for electric power distribution for industrial plants, ANSI/IEEE Std. 141-1993 (Red Book).

IEEE. (2010). Standard definitions for the measurement of electric power quantities under sinusoidal, nonsinusoidal, balanced or unbalanced conditions. STD 1459-2010.

IEEE. (2014). Recommended practice and requirements for harmonic control in electric power systems. IEEE Std 519-2014 (Revision of IEEE Std 519-1992).

IEEE P519. (2015). IEEE draft guide for applying harmonic limits on power systems. IEEE P519.1/D12, 1-124.

Jamehbozorg, A., \& Shahrtash, S. M. (2010). A decision-tree-based method for fault classification in single-circuit transmission lines. IEEE Transactions on Power Delivery, 25(4), 2190-2196.

Junior, A. M. G., Silva, V. V. R., Baccarini, L. M. R., \& Reis, M. L. F. (2014). Three-phase induction motors faults recognition and classification using neural networks and response surface models. Journal of Control, Automation and Electrical Systems, 25(3), 330-338.

Lieberman, D. G., Troncoso, R. J. R., Rios, R. A. O., Perez, A. G., \& Yepez, E. C. (2011). Techniques and methodologies for power quality analysis and disturbances classification in power systems: A review. IET Generation, Transmission \& Distribution, 5(4), 519-529.
Livani, H., \& Evrenosoglu, C. Y. (2013). A fault classification and localization method for three-terminal circuits using machine learning. IEEE Transactions on Power Delivery, 28(4), 2282-2290.

Monedero, I., Leon, C., Ropero, J., Garcia, A., Elena, J. M., \& Montano, J. C. (2007). Classification of electrical disturbances in real time using neural networks. IEEE Transactions on Power Delivery, 22(3), 1288-1296.

Moravej, Z., Pazoki, M., \& Khederzadeh, M. (2015). New patternrecognition method for fault analysis in transmission line with upfc. IEEE Transactions on Power Delivery, 30(3), 1231-1242.

Moreira, A. C., Paredes, H. K. M., \& Silva, L. C. P. (2015). Applying conservative power theory for analyzing three-phase $\mathrm{X}$-ray machine impact on distribution systems. Elsevier Electric Power Systems Research, 129, 114-125.

NEMA. (1993). Motors and generators. NEMA Standards Publication no. MG1-1993.

Palácios, R. H. C., Goedtel, A., Godoy, W. F., \& Fabri, J. A. (2016). Fault identification in the stator winding of induction motors using pca with artificial neural networks. Journal of Control, Automation and Electrical Systems, 27(4), 406-418.

Ribeiro, P. F., Duque, C. A., Silveira, P. M., \& Cerqueira, A. S. (2013). Power systems processing for smart grids (1st ed.). Hoboken: Wiley.

Rodriguez, M. V., Troncoso, R. J. R., Rios, R. A. O., \& Perez, A. G. (2014). Detection and classification of single and combined power quality disturbances using neural networks. IEEE Transactions on Industrial Electronics, 61(5), 2473-2482.

Samantaray, S. R. (2009). Decision tree-based fault zone identification and fault classification in flexible ac transmissions-based transmission line. IET Generation, Transmission \& Distribution, 3(5), 425-436.

Samantaray, S. R. (2013). A data-mining model for protection of factsbased transmission line. IEEE Transactions on Power Delivery, 28(2), 612-618.

Santana, M. P., Monteiro, J. R. B. A., Borges, F. A. S., Paula, G. T., Almeida, T. E. P., Pereira, W. C. A., et al. (2017). Fault identification in doubly fed induction generator using $\mathrm{fft}$ and neural networks. Journal of Control, Automation and Electrical Systems, 28(2), 228-237.

Sokolova, M., \& Lapalme, G. (2009). A systematic analysis of performance measures for classification tasks. Elsevier Information Processing \& Management, 45(4), 427-437.

Statistics and Machine Learning Toolbox for MATLAB. (2017) MAT$L A B$ classification learner application mathworks. Retrieved October 03, 2017 from https://www.mathworks.com/help/stats/ classification-learner-app.html.

Tenti, P., Paredes, H. K. M., \& Matavelli, P. (2011). Conservative power theory, a framework to approach control and accountability issues in smart micro-grids. IEEE Transactions on Power Electronics, 26(3), 664-673.

Upadhyaya, S., Mohanty, S., \& Bhende, C. N. (2015). Hybrid methods for fast detection and characterization of power quality disturbances. Journal of Control, Automation and Electrical Systems, $26(5), 556-566$. 